\title{
Analysis of D-Shaped Optical Fiber based Corrosion Sensor Using LMR and SPR Effects
}

\author{
Valdemir M. da Silva Júnior ${ }^{1}$, Jehan F. Nascimento ${ }^{2}$, Joaquim F. Martins Filho ${ }^{10}$ \\ ${ }^{I}$ Department of Electronics and Systems, Federal University of Pernambuco, Recife, Brazil \\ valdemirjr01@gmail.com, joaquim.martins@ufpe.br \\ ${ }^{2}$ Interdisciplinary Nucleus of Nature and Exact Sciences, Federal University of Pernambuco, Caruaru, Brazil \\ jehan.nascimento@ufpe.br
}

\begin{abstract}
This article presents the proposed structure and the simulation results from analytical and numerical modeling of two corrosion sensor elements in D-shaped optical fiber: one based on the lossy mode resonance (LMR) effect and the other based on the effect of surface plasmon resonance (SPR). In the first sensor element, a bilayer of titanium dioxide - aluminum $\left(\mathrm{TiO}_{2}-\mathrm{Al}\right)$ is deposited on the D-shaped region, operating in LMR conditions, while, in the second sensor element, an aluminum (Al) monolayer is deposited under D-shaped region, operating in SPR condition. The sensor elements can operate separately, enabling simultaneous twoparameter measurements at two different points, or they can operate in cascade configuration, increasing the operating range and sensitivity of the sensor set. The D-shaped region of the optical fiber is modeled with an analytical model based on the Fresnel formulation, and also with a numerical model, which uses the finite element method with the COMSOL Multiphysics 5.2 software. The transmission of light through the D-shaped region causes peculiar variations in each light polarization in each sensor element, depending on the metal thickness. Both regions are subject to a corrosive environment. The sensor elements are evaluated separately and in cascade configuration, using polarized and nonpolarized light. Finally, the obtained results show two resonance valleys for the same operating wavelength, resulting in a higher operating range with high sensitivity, compared to other corrosion sensor structures found in the literature.
\end{abstract}

Index Terms - Cascaded Structure, Corrosion Sensor, D-shaped Optical Fiber Sensor, Lossy Mode Resonance, Surface Plasmon Resonance.

\section{INTRODUCTION}

In 2013, the annual global cost of corrosion impacts in industry and governments was estimated at more than $\$ 1.5$ trillion, equivalent to $3.4 \%$ of that year's gross global product [1] . Not including individual safety costs and environmental consequences, the estimated savings when using corrosion control processes is up to $35 \%$, that is, savings of up to US\$ 875 million [1]. These data justify and present the relevance of research and investments in devices that monitor and inhibit corrosion processes.

In particular, corrosion monitoring systems that use optical fiber sensors are considered good 
Journal of Microwaves, Optoelectronics and Electromagnetic Applications, Vol. 20, No. 3, September 2021

DOI: http://dx.doi.org/10.1590/2179-10742021v20i3254063

alternatives because they have several advantages, such as simplicity, safety, reliability, high sensitivity, in addition to other advantages of this category of sensors [2], [3]. Reviews about fiber optic corrosion sensors can be found in [4] and [5].

Among the sensors found in literature, four studies highlight the operation of the sensor based on variations in the intensity of the optical signal from the corrosion of the material deposited on the side of the optical fiber [6]-[9]. The configuration of the sensors [6]-[8] consists of a fiber optic region in which the cladding is chemically removed, and a metal layer is deposited on the side of the optical fiber core. In [6], the fiber used is a plastic-clad silica (PCS), combined with an electrochemical potential detection system to calculate the percentage of corroded metal in the acid solution around the sensor element. In [7], the optical fiber used is a multimode fiber (MMF), while in [8], a heterocore single-multi-single (SMS) structure is used, in which the multimode region has its cladding removed and a metal layer is deposited. The configuration used in [9] uses a Side-polished D-shaped single-mode fiber (SP-SMF), in which only the transverse magnetic (TM) polarization undergoes variation in intensity during the corrosion process, while the transverse electric (TE) component remains unchanged. In this way, this sensor operates as a polarizer, monitoring corrosion through the ratio of the TM and TE components at the end of the link.

In this work, it is proposed and evaluated the performance of two optical fiber corrosion sensor elements composed of regions of metallized D-shaped optical fiber, operating individually or in cascade, one in LMR condition, using the TE and TM polarizations, and the other in SPR condition, using only the TM polarization. The cascade configuration can present several resonance valleys occurring at different metal thickness, providing the combination of high sensitivity and wide operating range characteristics to the sensor. Moreover, the operation in two polarizations also allows the measurement in two different points, one for each polarization, or the measurement of two different parameters, corrosion and temperature, for example. In this work, emphasis is given on the increase in the sensor's operating range, together with a high sensitivity sensor characteristic, due to the existence of two resonance valleys, which can also be considered as warning stages of different levels of corrosion, facilitating the monitoring process. To the best of our efforts, no reference could be found in literature to the use of the LMR effect to measure corrosion, and there is no mention to the use of the SPR and LMR effects simultaneously in a single sensor device.

This work is an extended version of an article published in the MOMAG 2020 conference. In addition, to reinforcing the results previously presented with numerical simulations using the COMSOL Multiphysics software, we included the analysis of the sensor element response by taking the ratio of the TE/TM polarization signals, and the analysis of the sensor performance using nonpolarized light.

The structure of the first sensor element consists of a D-shaped optical fiber, on which a bilayer of $\mathrm{TiO}_{2}-\mathrm{Al}$ is deposited in order to satisfy the LMR conditions for the wavelength of interest 
Journal of Microwaves, Optoelectronics and Electromagnetic Applications, Vol. 20, No. 3, September 2021

DOI: http://dx.doi.org/10.1590/2179-10742021v20i3254063

$(\lambda=1550 \mathrm{~nm})$. This wavelength is chosen because of its low attenuation inthe standard silica optical fiber used, and because it is compatible with optical amplifiers and other standard fiber devices, allowing the use of several devices in a long-range multipoint sensor system as in [9]. The second sensor element is composed of a region of D-shaped fiber metallized only with aluminium (Al), satisfying the conditions for the SPR effect. Immersed in a corrosive environment, the variation of the metal (Al) thickness alters the conditions of light coupling for the LMR and SPR effects in the Dshaped regions, generating a peculiar relationship between the thickness of the metal and the intensity of the transmitted optical signal for each polarization of each sensor region, which is analyzed by attenuated total reflectance (ATR).

In addition to the cascade structure, it is possible to use the two sensor elements individually, using the LMR region to measure corrosion and the SPR region to measure another important parameter for the context of hostile environments, such as temperature, for example. The performance of the sensor is evaluated analytically using a model based on the Fresnel equations and also numerically, using the finite element method (FEM) with the COMSOL Multiphysics 5.2 software.

Section II presents a brief description of the fundamentals of the LMR effect and the use of this effect for optical fiber sensors, and also a comparison between the effects of LMR and SPR. Section III presents the analytical and numerical modeling used to simulate the D-shaped region of the optical fiber as sensor elements. Section IV presents the results and discussions. Finally, Section V brings the final considerations of this work.

\section{LMR AND SPR EFFECTS}

Since the first time it was reported in the construction of optical fiber sensors in 2010, by del Villar et al. [11], several experimental evidences of the potential of sensors based on the LMR effect have been published [12]-[15]. Similar to the SPR effect, the Kretschmann configuration can be used to excite the LMR effect, in general, with a thin film of polymers or metal oxides, such as indium-tin oxide (ITO), titanium dioxide $\left(\mathrm{TiO}_{2}\right)$, indium oxide $\left(\mathrm{In}_{2} \mathrm{O}_{3}\right)$, tantalum pentoxide $\left(\mathrm{Ta}_{2} \mathrm{O}_{5}\right)$, zinc oxide ( $\mathrm{ZnO})$, poly (allylamine hydrochloride) (PAH)/poly (styrene sulfonate) (PSS), among others [12-16]. Unlike the SPR effect, which occurs when the real part of the film's electric permittivity is negative and greater in modulus than the imaginary parts of the film and of the external medium electric permittivities, the LMR effect occurs when the real part of the film's electrical permittivity of the material is positive and larger in modulus than the imaginary parts of the electric permittivities of the thin film and the external medium [14], resulting in a wide variety of materials that can be used to compose the thin film.

The devices that operate on the basis of the LMR effect are similar to those that operate on the basis of the SPR effect, including the optical fiber sensor configurations [14], [15]. Both depend on the refractive index of the external medium and on the thickness of the thin films [14]. 
Journal of Microwaves, Optoelectronics and Electromagnetic Applications, Vol. 20, No. 3, September 2021 DOI: http://dx.doi.org/10.1590/2179-10742021v20i3254063

Despite being less popular in the scientific community, the LMR effect has advantages over the SPR effect, such as not needing noble metals, such as the SPR effect, and not being limited to the TM component to generate resonance. In the LMR effect the resonance can be observed in the polarizations $\mathrm{TM}$ and $\mathrm{TE}$ [15]. With respect to the excitation angle, when operated in the Kretschmann configuration, in general, the SPR effect is excited in a range between $40^{\circ}$ and $70^{\circ}$, while the LMR effect is typically excited at angles close to $90^{\circ}$, which can be better combined with optical fiber devices [15]. In terms of its spectral characteristics, devices that use the LMR effect also have an advantage over devices that use the SPR effect, since it is possible to excite multiple orders of the LMR as a function of the wavelength and polarization of light, while there is only an optimum point to excite SPR [12]-[15]. Two examples of the potential of LMR effect-based sensors are found in [16] and [17], in which a refractive index sensor using the LMR effect is more sensitive than the SPR effect for the same configuration.

Table I presents a summary of the characteristics of the SPR and LMR effects, which allows one to verify the difference and potential of the sensors based on the LMR and SPR effects.

TABLE I. PARAMETERS COMPARISON BETWEEN LMR AND SPR DEVICES, ADAPTED FROM [15].

\begin{tabular}{|c|c|c|}
\hline Parameters & SPR & LMR \\
\hline Polarization of light & TM polarized & $\mathrm{TM}$ and TE polarized light \\
\hline $\begin{array}{l}\text { Material Permitivity } \\
\qquad\left(\varepsilon=\varepsilon_{\mathrm{r}}+\mathrm{i} \varepsilon_{\mathrm{i}}\right)\end{array}$ & $\begin{array}{c}\varepsilon_{\mathrm{r}} \text { negative and higher in magnitude than both the } \varepsilon_{\mathrm{i}} \\
\text { and the surrounding medium. }\end{array}$ & $\begin{array}{l}\varepsilon_{\mathrm{r}} \text { positive and higher in magnitude than } \\
\text { both the } \varepsilon_{\mathrm{i}} \text { and the surrounding medium. }\end{array}$ \\
\hline Thin film materials & Metals, Ag (silver) and Au (gold). & $\begin{array}{l}\text { Metal oxides and polymers (wide range } \\
\text { of materials). }\end{array}$ \\
\hline Thin film thickness & No multiple SPRs with increase in thickness. & $\begin{array}{l}\text { Multiple LMRs can be generated with } \\
\text { increase in thickness }\end{array}$ \\
\hline Sensitivity & Maximum for a particular thickness of coating layer. & $\begin{array}{l}\text { Can be tuned just changing thickness of } \\
\text { the coating layer. }\end{array}$ \\
\hline
\end{tabular}

In the review articles about optical fiber sensors using LMR of 2015 [15], and 2018 [15], several chemical, biological and physical applications are reported, but no application in corrosion sensors were mentioned. Other sources in the literature were searched, but no LMR based corrosion sensor could be found.

In this article, the characteristics of the LMR effect to operate with two resonance valleys, one for the TM polarization and the other for the TE polarization, is explored, increasing the application possibilities, either by measuring two distinct points (one for each polarization in the configuration in cascade), using two critical levels of corrosion levels (resonance valleys), measuring two distinct parameters (one for each polarization), using a self-referenced system (from the polarization ratio) or using non-polarized light, which results in a simple and less expensive system compared to systems that require polarization control.

\section{DEVICE MODELING}

In this section, the computational models used to simulate the corrosion sensor elements are 
Journal of Microwaves, Optoelectronics and Electromagnetic Applications, Vol. 20, No. 3, September 2021 DOI: http://dx.doi.org/10.1590/2179-10742021v20i3254063

described. The first model is analytical and based on the Fresnel equations. It approximates the Dshaped optical fiber with the deposited thin films to a semi-infinite multilayer structure. The second model, numerical, uses the finite element method with the COMSOL Multiphysics 5.2 in a $2 \mathrm{D}$ geometry that represents the longitudinal section of the D-shaped optical fiber sensor structure.

\section{A. Analytical Model}

The analytical modeling of the sensor elements is based on the Fresnel equations applied to the sensor structure shown in Fig. 1, which presents the longitudinal section of the D-shaped optical fiber with core radius $r_{\text {core }}$, uniformly metallized with a bilayer of $\mathrm{TiO}_{2}-\mathrm{Al}$, with oxide and metal thicknesses $d_{o}$ and $d_{m}$, respectively, along a D-shaped region of length $L$, and a residual cladding thickness $d_{c}$ under the D-shaped region. In this model, the optical fiber sensor structure is approximated to a multilayer structure [18] with five materials of different refractive index (i = 1-core, 2-cladding, 3-oxide, 4-metal, 5-external medium). An optical signal with wavelength of $1550 \mathrm{~nm}$ propagates through the core of the optical strip. When passing through the D-shaped region, the light interacts with the sensitive area, where part of the light is absorbed at the oxide-metal and metal-external media interfaces.

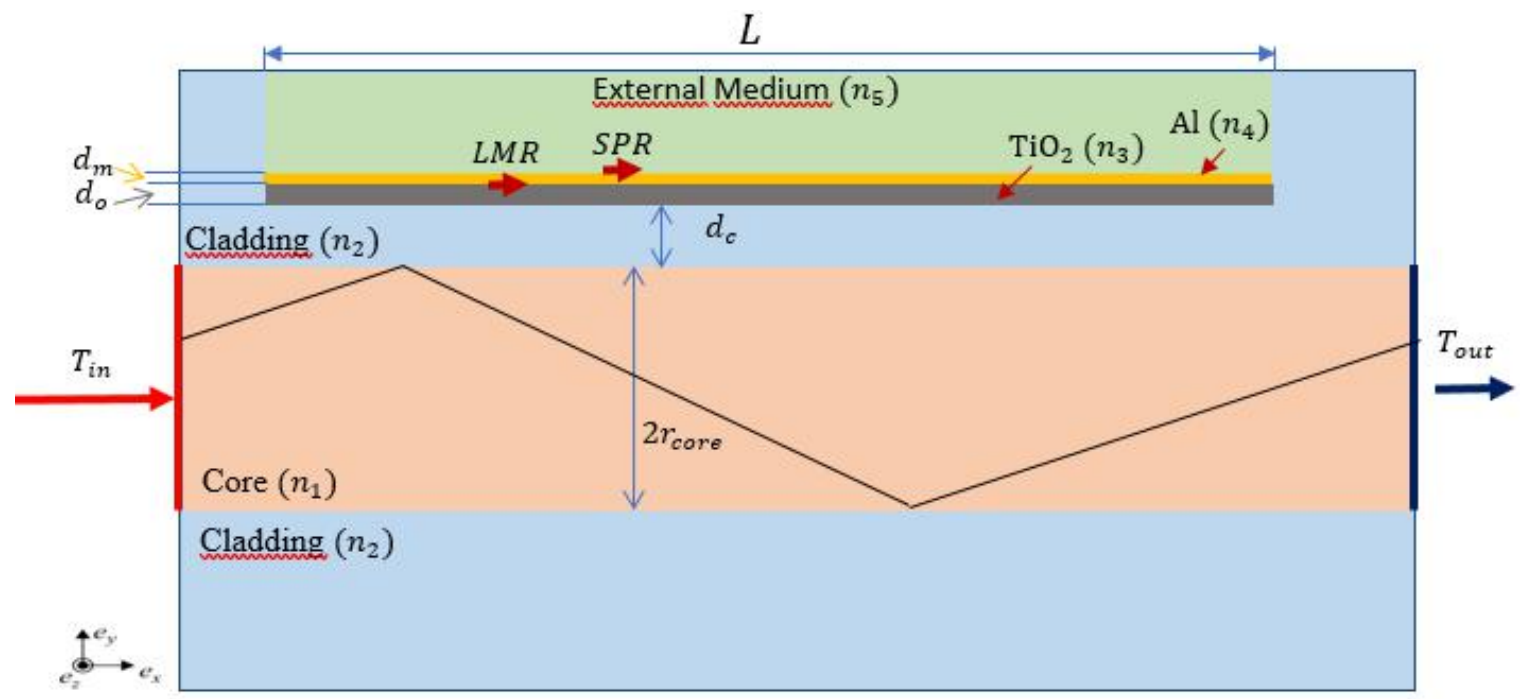

Fig. 1. Representation of the longitudinal section of the D-shaped region of the optical fiber sensor element $1 . \mathrm{L}$ represents the length of the sensor region. $\mathrm{r}_{\text {core }}, \mathrm{d}_{\mathrm{c}}, \mathrm{d}_{\mathrm{o}}$ and $\mathrm{d}_{\mathrm{m}}$ represent, respectively, core radius, residual cladding thickness in the D-shaped region, oxide thickness and metal thickness.

The normalized light transmission after passing through the D-shaped can be calculated using [19]:

with

$$
\mathrm{T}_{\mathrm{j}}=\frac{\int_{\theta_{\mathrm{cr}}}^{90^{\circ}} \mathrm{R}_{\mathrm{j}}^{\rho(\theta)} \mathrm{P}(\theta) \mathrm{d} \theta}{\int_{\theta_{\mathrm{cr}}}^{90^{\circ}} \mathrm{P}(\theta) \mathrm{d} \theta},
$$

$$
P(\theta)=\frac{n_{1}^{2} \sin \theta \cos \theta}{\left(1-n_{1}^{2} \cos ^{2} \theta\right)^{2}}
$$


Journal of Microwaves, Optoelectronics and Electromagnetic Applications, Vol. 20, No. 3, September 2021

DOI: http://dx.doi.org/10.1590/2179-10742021v20i3254063

and

$$
\mathrm{R}_{\mathrm{j}}=\left|\mathrm{r}_{\mathrm{j}}\right|^{2}
$$

$$
\theta_{\mathrm{cr}}=\sin ^{-1}\left(\mathrm{n}_{2} / \mathrm{n}_{1}\right) \text {, }
$$

where $n_{1}$ and $n_{2}$ are refractive indexes of medium 1 and 2 , respectively, $j=p$ the TM polarization and $\mathrm{j}=\mathrm{s}$ for the TE polarization. $\mathrm{P}(\theta)$ is the power distribution for the angle $\theta$, and $\theta_{\mathrm{cr}}$ is the critical angle. The number of attenuated reflections in the D-shaped region $\rho(\theta)$ is given by [20]:

$$
\rho(\theta)=\frac{\mathrm{L}}{2 \mathrm{r}_{\operatorname{core}} \tan \theta} \text {. }
$$

In (1) and (3), $R_{j}$ and $r_{j}(j=p$ or $s)$ are the reflectance and Fresnel reflection coefficient for the polarizations $\mathrm{TM}(\mathrm{j}=\mathrm{p})$ and $\mathrm{TE}(\mathrm{j}=\mathrm{s})$, respectively [21]. The values and parameters used in this study are found in Table II.

It is possible to operate with two metallized regions in the same cascade link, placing two D-shaped regions in sequence, with different configurations $[19,22]$. The total power after the passage of light can be calculated using the expression

$$
\mathrm{T}_{\mathrm{j}}=\mathrm{T}_{1_{\mathrm{j}}} \mathrm{T}_{2 \mathrm{j}},
$$

where $T_{1_{j}}$ and $T_{2 j}$ are the independent transmissions from sensor regions 1 and 2 , for polarization $\mathrm{j}$. In this article, two sensor elements that measure corrosion are proposed, the first operating under LMR conditions and the second operating under SPR conditions. The sensor element 2 is similar to the sensor element 1 , but without the deposition of $\mathrm{TiO}_{2}$, being considered a system of only 4 layers.

TABLE II. PARAMETERS USED IN SIMULATIONS OF THE SENSOR ELEMENTS.

\begin{tabular}{cccc}
\hline Parameter & Symbol & Value & Ref. \\
\hline Operating wavelength & $\lambda$ & $1550 \mathrm{~nm}$ & - \\
Core refractive index & $\mathrm{n}_{1}$ & 1.4503 & {$[20]$} \\
Cladding refractive index & $\mathrm{n}_{2}$ & 1.4422 & {$[20]$} \\
Oxide refractive index $\left(\mathrm{TiO}_{2}\right)$ & $\mathrm{n}_{3}$ & 2.4532 & {$[20]$} \\
Metal refractive index (Al) & $\mathrm{n}_{4}$ & $0.4598+\mathrm{j} 14.882$ & {$[20]$} \\
Core radius & $\mathrm{n}_{5}$ & 1.38 & {$[23]$} \\
Cladding radius & $\mathrm{r}_{\text {core }}$ & $62.5 \mu \mathrm{m}$ & - \\
Refractive index of corrosive medium (Al-Etcher) & $\mathrm{r}_{\text {clad }}$ & $1 \mathrm{~mm}$ & - \\
Length of the D-shaped region & $\mathrm{L}$ & $2 \mu \mathrm{m}$ & - \\
Residual cladding thickness in the D-shaped region & $\mathrm{d}_{\mathrm{c}}$ & $255 \mathrm{~nm}$ & - \\
Oxide thickness on the D-shaped region of the sensor & $\mathrm{d}_{\mathrm{o}}$ & & - \\
element 1 & & $40 \mathrm{~nm}$ &
\end{tabular}

The corrosion sensor elements operate through the ATR method, in which changes in the metal thickness change the LMR/SPR conditions for TE and TM polarizations, resulting in changes in the light intensity transmitted by the link after the light passes through the D-shaped regions.

A simpler sensor configuration employs unpolarized light passing through and sensor elements. It is simpler in experimental terms because it does not need polarization control equipment and devices. This condition can be obtained in the models by considering the average between the normalized 
Journal of Microwaves, Optoelectronics and Electromagnetic Applications, Vol. 20, No. 3, September 2021

DOI: http://dx.doi.org/10.1590/2179-10742021v20i3254063

transmissions of each polarization, since the unpolarized light has a distribution of light intensity of $50 \%$ TM and 50\% TE [15].

\section{B. Numerical Model}

The numerical modeling of sensor elements 1 and 2 was made using the finite element method (FEM) with the COMSOL Multiphysics 5.2 [24]. This software allows to simulate the sensor element considering a longitudinal 2D section of the D-shaped region of the optical fiber, as shown in Fig 2. The 2D simulation structure generates a satisfactory response when compared to experimental results for optical corrosion sensors [25] and refractive index sensors with D-shaped optical fibers [26].

Figure 2(a) presents the 2D geometry of the sensor element 1, based on the LMR effect, simulated in the COMSOL Multiphysics software, showing the longitudinal section of the D-shaped optical fiber metallized with the bilayer $\mathrm{TiO}_{2}-\mathrm{Al}$, in which the $\mathrm{TiO}_{2}$ film has thickness $\mathrm{d}_{\mathrm{o}}$ and refractive index $\mathrm{n}_{3}$, whereas the $\mathrm{Al}$ film has refractive index $\mathrm{n}_{4}$ and initial thickness of $\mathrm{d}_{\mathrm{m}}$. The D-shaped region is surrounded by a corrosive external medium of refractive index $\mathrm{n}_{5}$. The optical fiber has a radius $r_{\text {core }}$ and cladding $r_{\text {clad }}$. Figure 2(b) is similar to Fig. 2(a), but without the layer $\mathrm{TiO}_{2}$, and shows the 2D geometry of the sensor element 2, based on the SPR effect.

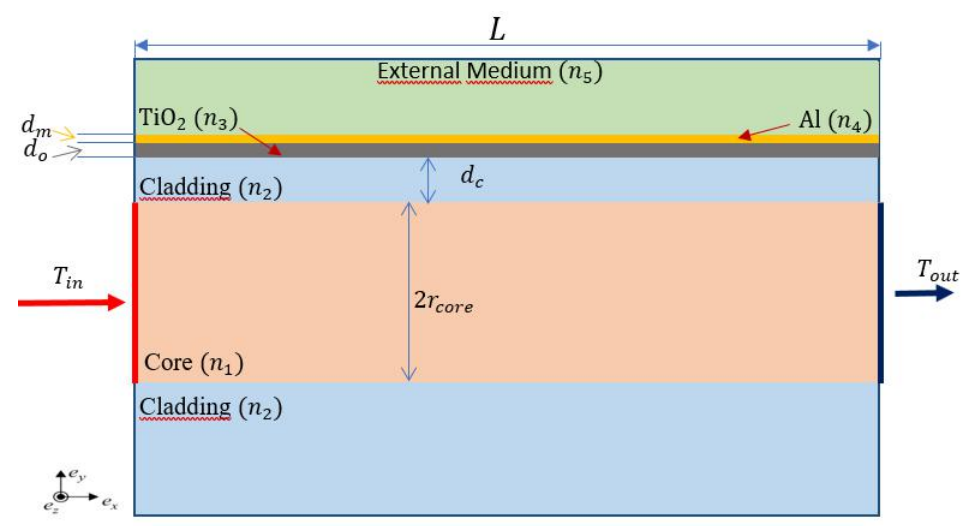

(a)

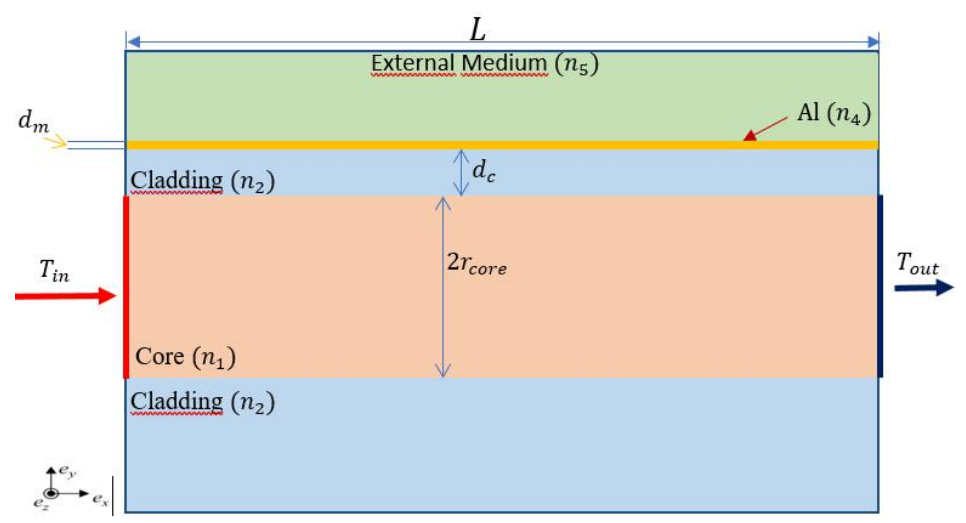

(b)

Fig. 2.Representation of the longitudinal section of the D-shaped optical fiber simulated in 2D using COMSOL Multiphysics for (a) sensor element 1 and (b) sensor element 2.

A beam with Gaussian profile is used to transmit an electromagnetic wave through the 2D structure, since it represents a good approximation for the fundamental mode that propagates in a single-mode Brazilian Microwave and Optoelectronics Society-SBMO received 31 Jan 2021; for review 31 Jan 2021 ; accepted 26 Apr 2021 Brazilian Society of Electromagnetism-SBMag $\quad$ C 2021 SBMO/SBMag $\quad$ (cc)) BY 
optical fiber [27]. The Gaussian profile beam is produced using a scattering boundary condition on the left edge of the optical fibers in Fig. 2, with a shape given by [28]

$$
\tilde{\mathrm{E}}_{0}=\left(\begin{array}{c}
\mathrm{E}_{0}^{\mathrm{x}} \\
\mathrm{E}_{0}^{\mathrm{y}} \\
\mathrm{E}_{0}^{\mathrm{z}}
\end{array}\right)=\left(\begin{array}{c}
0 \\
\cos \alpha \exp \left[-\left(\frac{y}{w_{0}}\right)^{2}\right] \\
\sin \alpha \exp \left[-\left(\frac{y}{w_{0}}\right)^{2}\right]
\end{array}\right),
$$

where $\mathrm{E}_{0}^{\mathrm{x}}, \mathrm{E}_{0}^{\mathrm{y}}$ and $\mathrm{E}_{0}^{z}$ are the components $\mathrm{x}, \mathrm{y}$ e $\mathrm{z}$ of the electric field that propagates in the optical fiber, respectively, $\alpha$ is the polarization angle of the electromagnetic wave and $\mathrm{w}_{0}$ is the width of the modeled Gaussian beam, considering the radius of the optical fiber. As the responses for the TE and TM polarizations are desired, $\alpha=45^{\circ}$ was adopted in order to obtain responses for TE and TM within the same simulation. However, as the results presented in Section IV are normalized, the same results can be obtained by calculating them separately for $\alpha=0^{\circ}(\mathrm{TM})$ and $\alpha=90^{\circ}$ (TE).

The light transmission at the right end of the D-shaped optical fiber core shown in Fig. 1 is calculated using a transmission coefficient, obtained from parameter $S_{21}$ [24]. The total transmission of light passing through the D-shaped region is given by [24, 28]:

$$
\mathrm{T}_{\text {out }}=\left\|\mathrm{S}_{21}\right\|^{2}=\left\|\mathrm{S}_{21}^{\mathrm{TM}}\right\|^{2}+\left\|\mathrm{S}_{21}^{\mathrm{TE}}\right\|^{2} \Rightarrow \mathrm{T}_{\text {out }}=\frac{\int\left\|\mathrm{E}_{\mathrm{L}}^{\mathrm{y}}\right\|^{2} \mathrm{dz}}{\int\left\|\widetilde{\mathrm{E}}_{0}\right\|^{2} \mathrm{dz}}+\frac{\int\left\|\mathrm{E}_{\mathrm{L}}^{\mathrm{z}}\right\|^{2} \mathrm{dz}}{\int\left\|\widetilde{\mathrm{E}}_{0}\right\|^{2} \mathrm{dz}},
$$

where $\mathrm{E}_{\mathrm{L}}^{\mathrm{y}}$ and $\mathrm{E}_{\mathrm{L}}^{\mathrm{z}}$ are the components of the electric field measured by the COMSOL Multiphysics software at the end of the D-shaped region for TM and TE polarization, respectively.

The normalized transmission for each polarization is given by

$$
\mathrm{T}_{k}=\left\|\mathrm{S}_{21}^{k}\right\|^{2}=\frac{\int\left\|\mathrm{E}_{\mathrm{L}}^{k}\right\|^{2} \mathrm{dz}}{\int\left\|\mathrm{E}_{0}^{k}\right\|^{2} \mathrm{dz}}
$$

where $\mathrm{k}=\mathrm{y}$ for $\mathrm{TM}$ polarization and $\mathrm{k}=\mathrm{z}$ for TE polarization. The results for the sensor elements 1 and 2 in Section IV were calculated using (1) for the analytical modeling and (9) for the numerical modeling. Equation (6) was used for the cascade structure for both models.

\section{RESULTS AND DISCUSSIONS}

\section{A. Sensor Element 1 - LMR}

Fig. 3 presents the result for the light transmission in the sensor element 1 as a function of the thickness of the aluminum, for the configuration described in Section III, where the TE and TM polarizations are analyzed separately. The sensor measures the variation in the thickness of the aluminum through the intensity of the light transmitted after passing through the D-shaped region. It is possible to observe that the sensor element based on the LMR effect can operate in different bands, with resonance valleys in $4.5 \mathrm{~nm}$ for TM polarization and $13 \mathrm{~nm}$ for TE polarization. Both valleys can operate as corrosion alert stages.

Brazilian Microwave and Optoelectronics Society-SBMO received 31 Jan 2021; for review 31 Jan 2021 ; accepted 26 Apr 2021 


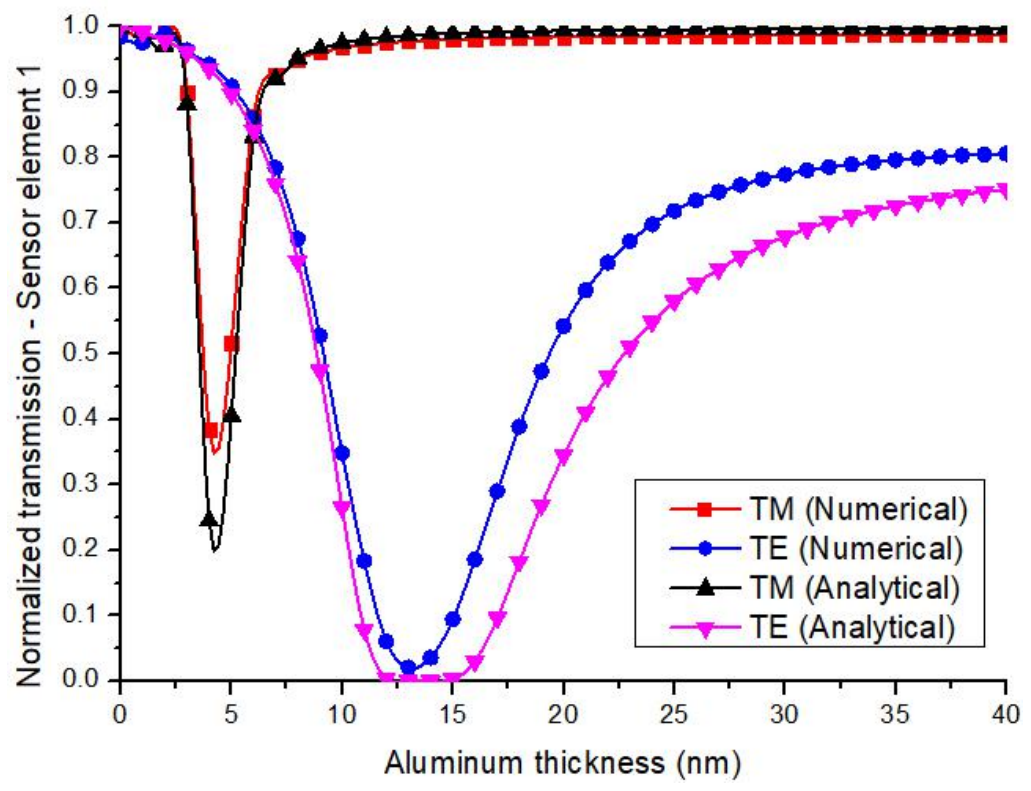

Fig. 3. Normalized light transmission for the TE and TM polarizations through the sensor element 1 as a function of the thickness of the metallic film, designed with the parameters presented in Table II.

The curves of normalized light transmission as a function of the aluminum thickness for the sensor element 1 of Fig. 3 show that different resonance regions are obtained for each polarization, as it is a characteristic of the LMR effect. For TE polarization it is possible to measure corrosion in the operating range between $5 \mathrm{~nm}$ and $22 \mathrm{~nm}$ with resonance valley centered at $13 \mathrm{~nm}$, while for TM polarization it is possible to measure corrosion with high sensitivity in the operating range between 3 $\mathrm{nm}$ and $6 \mathrm{~nm}$, with a resonance valley centered at $4.5 \mathrm{~nm}$. In this way, the sensor element 1 allows to measure corrosion in a long operating range, with high sensitivity and with two resonance valleys in a single sensor element.

Defining corrosion sensor sensitivity as the modulus of the variation of light transmission divided by the variation of the metal thickness, high sensitivities are obtained for both polarizations in sensor element 1, as shown in Table III. The analytical and the numerical models results shown in Fig. 3 are in good agreement and they present similar sensitivities and operating ranges for the sensor element 1. The sensor element 1, alone, can measure corrosion in a total operating range of $19 \mathrm{~nm}$, between 3 and $22 \mathrm{~nm}$, with two resonance valleys, resulting in high sensitivities.

For the sake of comparison, the corrosion sensor that uses the SPR effect presented by Alves et al. [25] reached a sensitivity of $0.06 / \mathrm{nm}$, obtained for the TM polarization in the $5 \mathrm{~nm}$ operating range, between $7.5 \mathrm{~nm}$ and $12.5 \mathrm{~nm}$ [25]. Whereas the results obtained for the TM polarization in the LMR effect corrosion sensor presented in Table III show a sensitivity approximately 8.8 times greater in the range $3 \mathrm{~nm}-4.5 \mathrm{~nm}$, considering the analytical model. For the numerical model, the sensitivity is 7 times greater in the range $3 \mathrm{~nm}-4.5 \mathrm{~nm}$.

Regarding the sensor range of operation, the sensor proposed by Alves et al. [25] has a useful operating range of $13 \mathrm{~nm}$ with resonance valley centered at $7 \mathrm{~nm}$, while the sensor element 1 
Journal of Microwaves, Optoelectronics and Electromagnetic Applications, Vol. 20, No. 3, September 2021

DOI: http://dx.doi.org/10.1590/2179-10742021v20i3254063

proposed in this article has a useful operating range of $22 \mathrm{~nm}$ with two valleys centered at $4.5 \mathrm{~nm}$ and $13 \mathrm{~nm}$ for the TM and TE polarizations, respectively.

TABLE III. SENSITIVITIES AND OPERATING RANGES FOR THE SENSOR ELEMENT 1.

\begin{tabular}{cccc}
\hline Model & Polarization & Operating range & Sensitivity Module \\
\hline Analytical & TM & $3 \mathrm{~nm}-4.5 \mathrm{~nm}$ & $0.53 / \mathrm{nm}$ \\
Numerical & TM & $3 \mathrm{~nm}-4.5 \mathrm{~nm}$ & $0.43 / \mathrm{nm}$ \\
Analytical & TM & $4.5 \mathrm{~nm}-6 \mathrm{~nm}$ & $0.46 / \mathrm{nm}$ \\
Numerical & TM & $4.5 \mathrm{~nm}-6 \mathrm{~nm}$ & $0.37 / \mathrm{nm}$ \\
Analytical & TE & $5 \mathrm{~nm}-12 \mathrm{~nm}$ & $0.13 / \mathrm{nm}$ \\
Numerical & TE & $5 \mathrm{~nm}-12 \mathrm{~nm}$ & $0.13 / \mathrm{nm}$ \\
Analytical & TE & $15 \mathrm{~nm}-22 \mathrm{~nm}$ & $0.09 / \mathrm{nm}$ \\
Numerical & TE & $15 \mathrm{~nm}-22 \mathrm{~nm}$ & $0.07 / \mathrm{nm}$ \\
\hline
\end{tabular}

\section{B. Sensor Element 2 - SPR}

Figure 4 shows the normalized transmission of light as a function of the aluminum thickness for the sensor element 2 designed with the configuration described in Section III for TE and TM polarizations separately. This configuration has no resonance valley in any of the polarizations and, being configured in SPR conditions, the TE polarization does not vary with the variation of the metal thickness during corrosion. However, it has a long operating range of up to $30 \mathrm{~nm}$, between $5 \mathrm{~nm}$ and $35 \mathrm{~nm}$, with lower sensitivities than in the LMR case, compatible with a long operating range, as can be seen in Table IV.

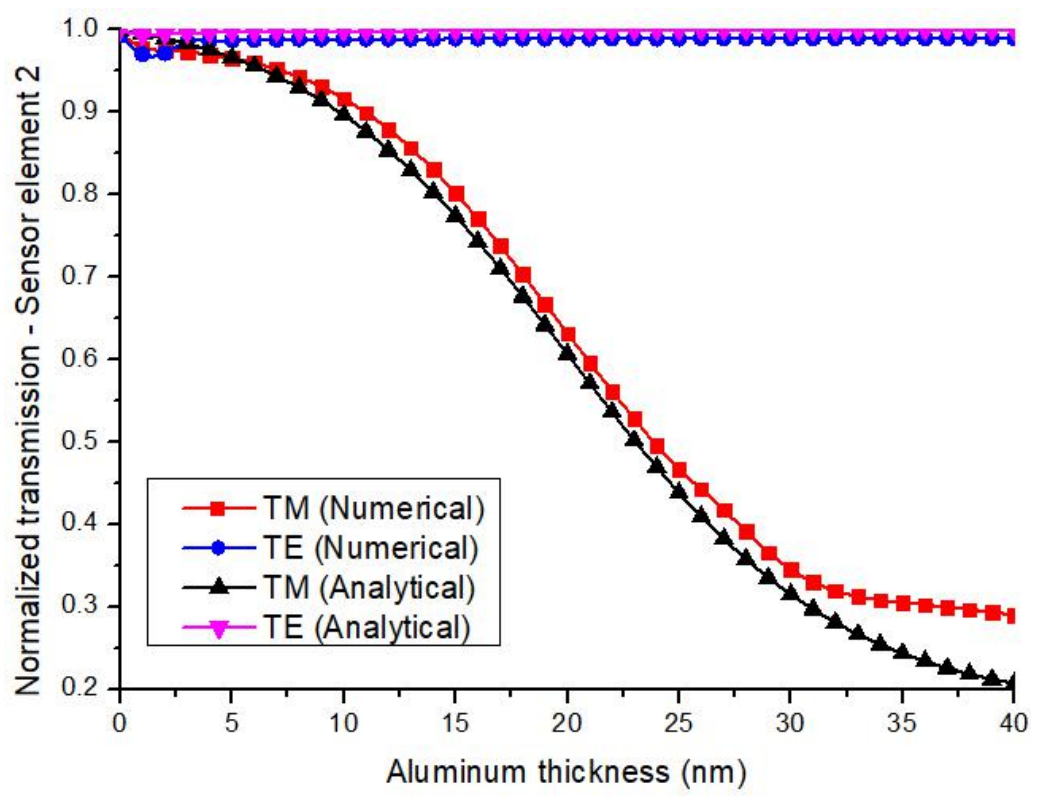

Fig. 4. Normalized light transmission for the TE and TM through the sensor element 2, designed with the parameters presented in Table II as a function of the aluminum thickness.

In Figures 3 and 4, there is a good agreement between the results obtained by the analytical and numerical models. The presented results accredit the sensor elements 1 and 2 to be cascaded, since the sensor element 1 presents high sensitivities, whereas the sensor element 2 has a long operating range between $5 \mathrm{~nm}$ and $35 \mathrm{~nm}$. 
Journal of Microwaves, Optoelectronics and Electromagnetic Applications, Vol. 20, No. 3, September 2021

DOI: http://dx.doi.org/10.1590/2179-10742021v20i3254063

TABLE IV. SENSITIVITIES AND OPERATING RANGES FOR THE SENSOR ELEMENT 2.

\begin{tabular}{cccc}
\hline Model & Polarization & Operating range & Sensitivity Module \\
\hline Analytical & TM & $5 \mathrm{~nm}-35 \mathrm{~nm}$ & $0.023 / \mathrm{nm}$ \\
Numerical & TM & $5 \mathrm{~nm}-35 \mathrm{~nm}$ & $0.018 / \mathrm{nm}$ \\
Analytical & TE & - & - \\
Numerical & TE & - & - \\
\hline
\end{tabular}

\section{Cascaded Structure of sensors elements 1 and $2-L M R$ and SPR}

Using a cascade system of D-shaped regions [19, 22], where the two sensor elements are fabricated along the same D-shaped optical fiber or, alternatively, they are fusion spliced close together, it is possible to measure different quantities or a quantity over a wider range of operation. In this article, cascading sensor elements 1 and 2 are considered, so that the cascade effect results in high sensitivity and ling operating range of the corrosion sensor.

Figure 5 shows the behavior of the sensor elements 1 and 2 in a cascade structure for the TE and TM polarizations using (6). The result shows that the cascade structure optimizes the performance of the TM polarization when compared to the response of the sensor elements 1 and 2 operating separately. This is because the cascade configuration maintains the resonance valley characteristic of the sensor element 1 in the TM polarization, at $4.5 \mathrm{~nm}$, and maintains the significant corrosion measurements up to $35 \mathrm{~nm}$, characteristic of the sensor element 2. For the TE polarization, the cascade structure response is basically a replica of the TE polarization response of sensor element 1 , since, in sensor element 2, TE polarization is insensitive to variations in metal thickness. The sensitivities are similar to those obtained in Tables III and IV in combination, since the TM polarization variations occur for different metal thickness in the sensor elements 1 and 2, and the TE polarization of the sensor element 2 is insensitive for the entire operating range.

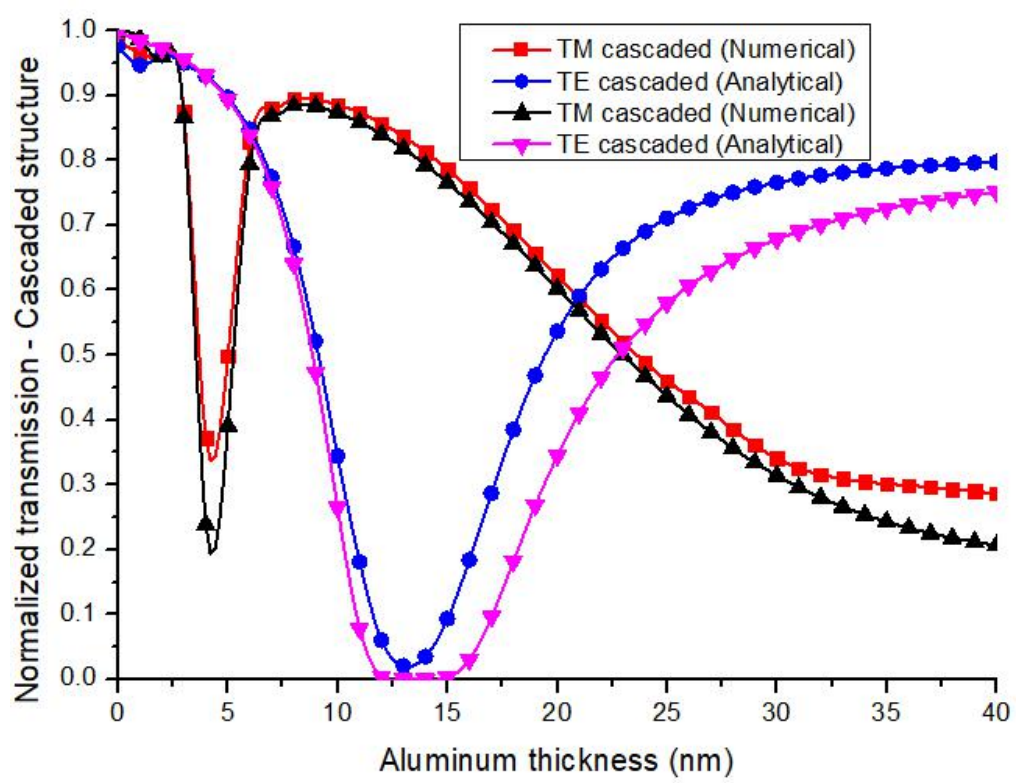

Fig. 5. Normalized light transmission for the TE and TM polarizations in a cascade configuration of the sensor elements 1 and 2, designed with the parameters presented in Table II as a function of the aluminum thickness.

Another possibility to analyze the cascade configuration is to adopt the procedure used in [9] , 
Journal of Microwaves, Optoelectronics and Electromagnetic Applications, Vol. 20, No. 3, September 2021 DOI: http://dx.doi.org/10.1590/2179-10742021v20i3254063

taking the ratio between the TE and TM components to evaluate the performance of the sensor composed by the cascading sensor elements 1 and 2, as shown in Fig. 6. As it can be seen, the sensor has a linear response between $15 \mathrm{~nm}$ and $35 \mathrm{~nm}$. Despite having a range without satisfactory response between $11 \mathrm{~nm}$ and $15 \mathrm{~nm}$, due to its TE transmission being close to zero, there are good responses in the interval between $3 \mathrm{~nm}$ and $11 \mathrm{~nm}$, showing a high peak at $4.5 \mathrm{~nm}$. The results for the sensitivities and operating ranges considered for the cascade sensor verified from the TE / TM ratio are shown in Table V.

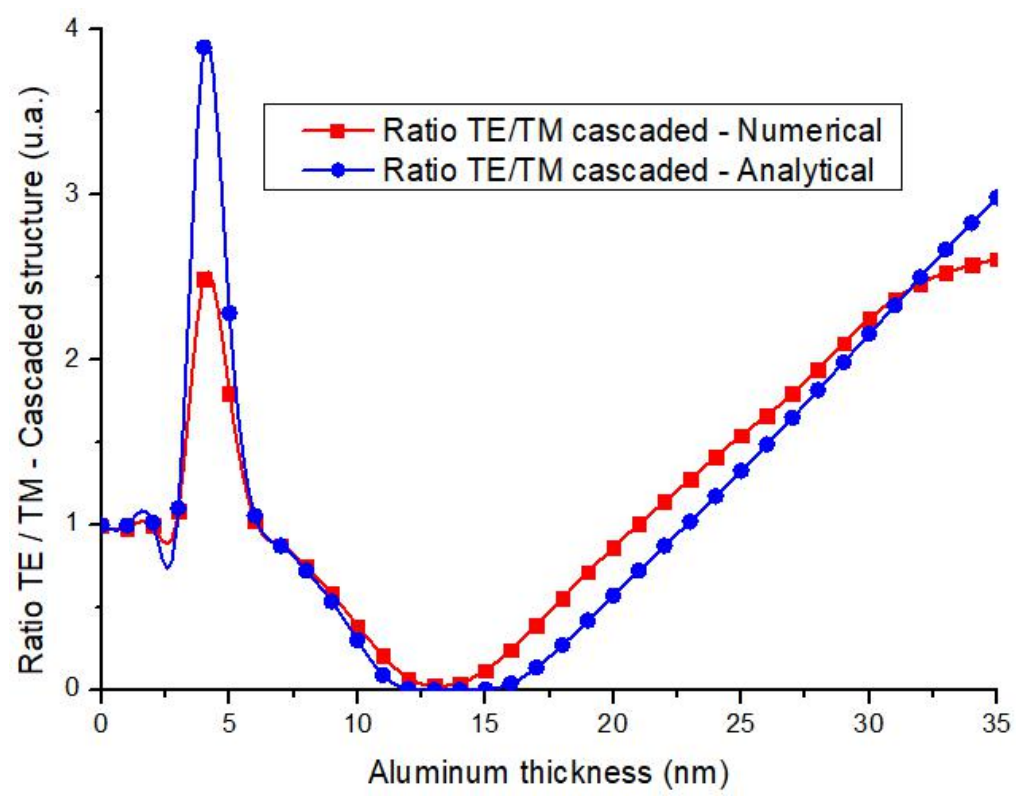

Fig. 6. TE / TM ratio for the cascaded structure configuration of the sensor elements 1 and 2, designed with the parameters presented in Table II as a function of aluminum thickness.

TABLE V. SENSITIVITY AND OPERATING RANGES FOR THE CASCADED STRUCTURE FOR THE RATIO TE/TM.

\begin{tabular}{cccc}
\hline Model & Ratio & Operating range & Sensitivity Module \\
\hline Analytical & TE/TM & $2.5 \mathrm{~nm}-4.5 \mathrm{~nm}$ and $4.5 \mathrm{~nm}-6 \mathrm{~nm}$ & $2 / \mathrm{nm}$ \\
Numerical & TE/TM & $2.5 \mathrm{~nm}-4.5 \mathrm{~nm}$ and $4.5 \mathrm{~nm}-6 \mathrm{~nm}$ & $1 / \mathrm{nm}$ \\
Analytical & TE/TM & $6 \mathrm{~nm}-11 \mathrm{~nm}$ & $0.2 / \mathrm{nm}$ \\
Numerical & TE/TM & $6 \mathrm{~nm}-11 \mathrm{~nm}$ & $0.2 / \mathrm{nm}$ \\
Analytical & TE/TM & $15 \mathrm{~nm}-35 \mathrm{~nm}$ & $0.15 / \mathrm{nm}$ \\
Numerical & TE/TM & $15 \mathrm{~nm}-35 \mathrm{~nm}$ & $0.13 / \mathrm{nm}$ \\
\hline
\end{tabular}

Another possibility for the analysis of the cascade sensor is to take the case that the light is not polarized. It is less expensive in terms of equipment, as it does not need polarization control systems and devices. The result obtained for non-polarized light is shown in Fig. 7, in which it is possible to observe the maintenance of the two resonance valleys, although presenting valleys of lower depth, since there is an equal composition between the TE and TM polarizations. Similar to the sensor element 1 operating in isolation, the cascade sensor with non-polarized light has a long operating range, from $3 \mathrm{~nm}$ to $22 \mathrm{~nm}$, with resonance valleys at $4.5 \mathrm{~nm}$ and $13 \mathrm{~nm}$.

The use of non-polarized light decreases the sensitivity of the cascade sensor, as can be seen in Table VI, mainly for metal thicknesses higher than $22 \mathrm{~nm}$. This is because it is in this region the TE 
Journal of Microwaves, Optoelectronics and Electromagnetic Applications, Vol. 20, No. 3, September 2021

DOI: http://dx.doi.org/10.1590/2179-10742021v20i3254063

and TM polarizations have close normalized transmission values, but with slopes of opposite signals, so that a variation in the intensity of the TM polarization compensates part of the variation in the intensity of the TE polarization.

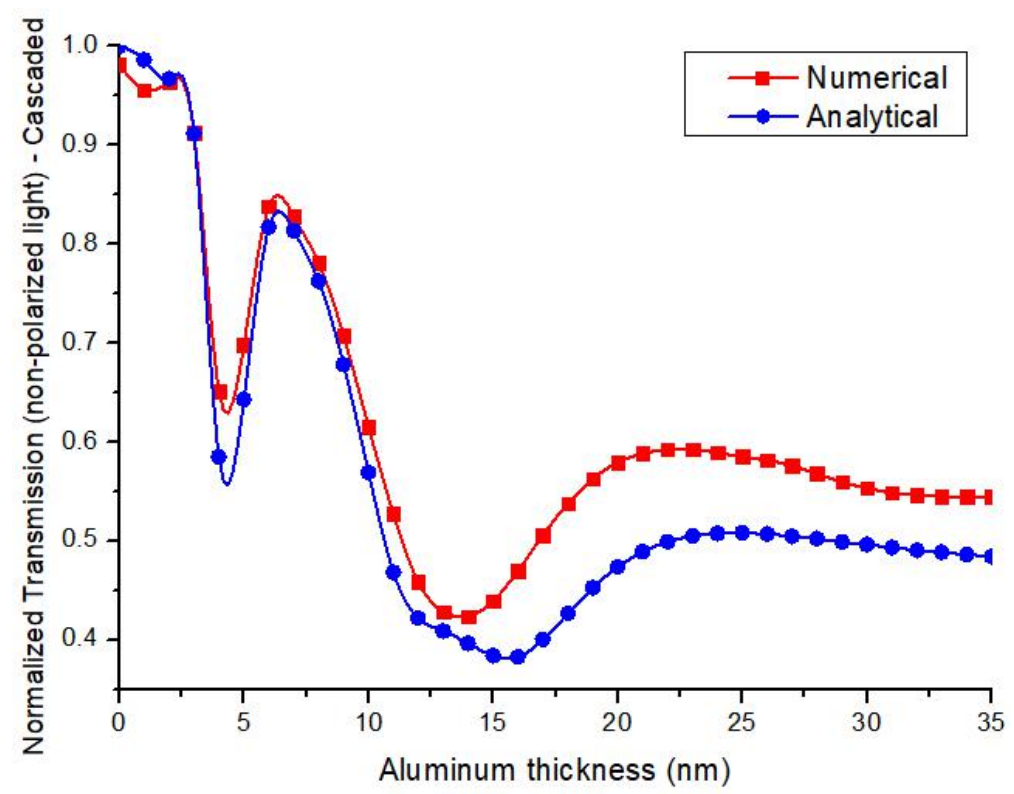

Fig. 7. Normalized transmission of non-polarized light for the cascade configuration of sensor elements 1 and 2, designed with the parameters presented in Table II as a function of the aluminum thickness.

TABLE VI. SENSITIVITIES AND OPERATING RANGES FOR THE CASCADED STRUCTURE FOR NON-POLARIZED LIGHT.

\begin{tabular}{c|c|c}
\hline Model & Operating range & Sensitivity Module \\
\hline Analytical & $3 \mathrm{~nm}-4.5 \mathrm{~nm}$ & $0.26 / \mathrm{nm}$ \\
Numerical & $3 \mathrm{~nm}-4.5 \mathrm{~nm}$ & $0.22 / \mathrm{nm}$ \\
Analytical & $4.5 \mathrm{~nm}-6 \mathrm{~nm}$ & $0.16 / \mathrm{nm}$ \\
Numerical & $4.5 \mathrm{~nm}-6 \mathrm{~nm}$ & $0.14 / \mathrm{nm}$ \\
Analytical & $6 \mathrm{~nm}-12 \mathrm{~nm}$ & $0.06 / \mathrm{nm}$ \\
Numerical & $6 \mathrm{~nm}-12 \mathrm{~nm}$ & $0.07 / \mathrm{nm}$ \\
Analytical & $15 \mathrm{~nm}-22 \mathrm{~nm}$ & $0.016 / \mathrm{nm}$ \\
Numerical & $15 \mathrm{~nm}-22 \mathrm{~nm}$ & $0.02 / \mathrm{nm}$ \\
\hline
\end{tabular}

\section{CONCLUSION}

In this article, the proposal and the results of numerical and analytical simulations of two configurations of corrosion sensors are presented. The sensors are based on D-shaped optical fiber and they can operate isolate or in a cascade structure. The first sensor element operates under LMR conditions, it has a bilayer of $\mathrm{TiO}_{2}-\mathrm{Al}$ on the D-shaped region and it has a response to TE and TM polarizations, resulting in two resonance valleys. The second sensor element operates out of SPR resonance condition and it has a monolayer of $\mathrm{Al}$. In the simulations of sensor operation, it is assumed that both sensors are immersed in the same corrosive liquid.

The sensor element 1, which uses the LMR effect, presents a resonance valley for each light polarization, with variations of more than $50 \%$ in the light intensity per $\mathrm{nm}$ of variation of metal thickness $(0.53 / \mathrm{nm}$ in the range $4.5-6 \mathrm{~nm})$ and an operating range of $19 \mathrm{~nm}$, between $3 \mathrm{~nm}$ and $22 \mathrm{~nm}$ of metal thickness. 
Journal of Microwaves, Optoelectronics and Electromagnetic Applications, Vol. 20, No. 3, September 2021

DOI: http://dx.doi.org/10.1590/2179-10742021v20i3254063

The sensor element 2, which uses the SPR effect, was designed to present no resonance valley. However, it has two important characteristics: a long operating range for the TM polarization of $30 \mathrm{~nm}$, between $5 \mathrm{~nm}$ and $35 \mathrm{~nm}$ of metal thickness, with sensitivity of $0.023 / \mathrm{nm}$, and TE polarization in sensitivity to the variation of the metal thickness.

The sensor composed of the two cascading D-shaped regions was also evaluated. For the cascade it is assumed that the two regions of D-shaped are close together, so that the polarizations of light are maintained between them. With the cascade structure, the response of the TM polarization has been optimized by bringing together the best characteristics of the sensor elements 1 and 2 for the TM polarization. For the cascading TE polarization, there are no significant changes in relation to the response of the sensor element 1 to the TE polarization, since the sensor element 2 is sensitive only to the TM polarization. For the TM polarization, the cascade structure presents the resonance valley at $4.5 \mathrm{~nm}$, coming from the sensor element 1 , and the long operating range of the sensor element 2.

For the cascade of sensor elements 1 and 2, the response was also evaluated from the ratio between the TE and TM polarizations light intensities, after the light passed through the two D-shaped regions. The analysis by the polarization ratio reveals a sensor with a long operating range, high sensitivity, a peak region and a blind region, close to $5 \mathrm{~nm}$ and $12 \mathrm{~nm}$, respectively. When analyzing the transmission of polarizations separately, the blind region can be considered an alert region. Moreover, the signal analysis using the TE/TM ratio in the cascade structurepresents a linear response of the sensor in the operating range from $15 \mathrm{~nm}$ to $35 \mathrm{~nm}$ of metal thickness, with a sensitivity of $0.15 / \mathrm{nm}$.

Still on the cascade structure, the performance of the sensor elements 1 and 2 in cascade was also evaluated for the case where the light that passes through the D-shaped regions is non-polarized. The results showed a lower sensitivity and shorter operating range of the sensor in this case, compared to the previous cases. However, from the practical implementation point of view, the use of nonpolarized light has advantages, since it does not require equipment to control polarization, as well as it does not require the use of polarization maintaining fibers, which makes the cascade structure with non-polarized light simpler and less expensive than the cascade structure with polarized light.

\section{REFERENCES}

[1] G. Koch, J. Varney, N. Thompson, O. Moghissi, M. Gould and J. Payer, International of Measures of Prevention, Application, and Economics of Corrosion Technologies Study, Houston: NACE International, 2016.

[2] J. F. Martins-Filho and E. Fontana, "Optical Fiber Sensor System for Multipoint Corrosion Detection", in Optical Fibre, New Developments, 1 ed., In-Tech, 2009, pp. 35-44.

[3] J. Haus, Optical Sensors: Basics and Applications, New York, NY: Wiley-VCH Verlag, 2010.

[4] S. A. Wade, C. D. Wallbrink, G. McAdam, S. Galea, B. R. W. Hinton and R. Jones, "A fibre optic corrosion fuse sensor using stressed metal-coated optical fibres", Sensors and Actuators B: Chemical, vol. 131, no. 2, pp. 602-608, 2008.

[5] R. F. Wirght, P. Lu, J. Devkota, F. Lu, M. Ziomek-Moroz and P. R. J. Ohodnicki, "Corrosion Sensors for Structural Health Monitoring of Oil and Natural Gas Infrastructure: A Review", Sensors, vol. 19, no. 3964, 13 Setembro 2019.

[6] M. Benounis and N. Jaffrezic-Renault, "Elaboration of a n optical fibre corrosion for aircraft applications", Sensors ana Actuators B, vol. 100, pp. 1-8, 2004.

[7] S. Dong, Y. Liao and Q. Tian, "Sensing of corrosion on aluminum surfaces by use of metallic optical fiber," Applied Optics, vol. 44, no. 30, pp. 6334-6337, 20 Outubro 2005.

[8] P. Y. Aisyah, A. M. Hatta and D. Y. Pratama, "Design of SMS (Single mode Multi mode Coreless - Single mode) 
Journal of Microwaves, Optoelectronics and Electromagnetic Applications, Vol. 20, No. 3, September 2021

DOI: http://dx.doi.org/10.1590/2179-10742021v20i3254063

Optical Fiber as Corrosion Sensor", in Second International Seminar on Photonics, Optics, and Its Applications, Bali, 2016.

[9] W. Hu, L. Ding, C. Zhu, D. guo, W. Yuan, N. Ma and W. Chen, "Optical Fiber Polarizer With Fe-C Film for Corrosion Monitoring"., IEEE Sensors Journal, vol. 17, no. 21, pp. 6904-6910, 2017.

[10] J. F. Nascimento, M. J. Silva, I. J. S. Coêlho, E. Cipriano, and J. F. Martins-Filho, "Amplified OTDR Systems for Multipoint Corrosion Monitoring," Sensors, vol. 12, no. 3, pp. 3438-3448, Mar. 2012.

[11] I. del Villar, C. R. Zamarreño, M. Hernaez and I. R. Matias, "Lossy mode resonance generation with indium-tin-oxidecoated optical fibers for sensing applications", Journal of Lightwave Technology, vol. 28, no. 1, pp. 111-117, 2010.

[12] F. J. Arregui, I. Del Villar, J. M. Corres, J. Goicochea, C. R. Zamarreño, ElosuaCesar, M. Hernaez, P. J. Rivero, A. B. Socorro, A. Urrutia, P. Sanchez, P. Zubiate, D. Lopez, N. De Acha and I. R. Matias, "Fiber-Optoc Lossy Mode Resonance Sensors", in Procedia Engineering, vol. 87, pp. 3-8, 2014.

[13] N. Paliwal and J. John, "Lossy Mode Resonance (LMR) Based Fiber Optic Sensors: A Review," in IEEE Sensors Journal, vol. 15, no. 10, pp. 5361-5371, Oct. 2015.

[14] I. Del Villar, M. Hernaez, C. R. Zamarreño, P. Sánchez, C. Fernández-Valdivieso, F. J. Arregui and I. R. Matias, "Design rules for lossy mode resonance based sensors", Applied Optics, vol. 51, no. 19, pp. 4298-4307, 2012.

[15] Q. Wang and W.-M. Zhao, "A comprehensive review of lossy mode resonance-based fiber optic sensors", Optics ana Lasers in Engineering, vol. 100, pp. 47-60, 2018.

[16] X.-Z. Wang and Q. Wang, "Theoretical Analysis of a Novel Microstructure Fiber Sensor Based on Lossy Mode Resonance", Electronics, vol. 8, no. 5, p. 484, 30 Abril 2019.

[17] S. Zhu, F. Pang, S. Huang, F. Zou, Y. Dong and T. Wang, "High sensitivity refractive index sensor based on adiabatic tapered optical fiber deposited with nanofilm by ALD", Optical Express, vol. 23, pp. 13880-13888, 2015.

[18] M.-H. Chiu, C.-H. Shih and M.-H. Chi, "Optimum sensitivity of single-mode D-type optical fiber", Sensors and Actuators, vol. 123, no. 2, pp. 1120-1124, Maio 2007.

[19] W. Yuan, L. Wang and J. Huan, "Theoretical investigation for two cascaded SPR fiber optic sensors", Sensors and Actuators B, vol. 161, no. 1, pp. 269-273, 2012.

[20] D. F. d. N. d. Santos, "Investigação Numérica do Desempenho de um Sensor de Fibra Óptica de Índice de Refração, Baseado em SPR e Utilizando COMSOL Multiphysics", Master's Dissertation (Master's Dissertation in Telecommunications Engineering and Power Networks) - UMA, Funchal, 2013.

[21] S. Li, L. Gao, C. Zou, W. Xie, Y. Wei, C. Tian, Z. Wang, F. Liang, Y. Xiang and Q. Yang, "A Polarization-Independent Fiber-Optic SPR Sensor", Sensors, vol. 18, no. 3204, 2018.

[22] Y. Lin, W. Tsai, Y. Tsao and J. Tai, "An Enhanced Optical Multimode Fiber Sensor Based on Surface Plasmon Resonance With Cascaded Structure", in IEEE Photonics Technology Letters, vol. 20, no. 15, pp. 1287-1289, Aug.1, 2008.

[23] E. A. Silva-Jr, A. P. C. da Cruz, J. F. Martins-Filho and J. N. Nascimento, "Sensor de Corrosão Utilizando Dupla Camada Metálica em Fibra Óptica", in $16^{a}$ SBMO - Simpósio Brasileiro de Micro-ondas e Optoeletrônica e $11^{a}$ CBMag - Congresso Brasileiro de Eletromagnetismo, Curitiba, Brazil, 2014.

[24] COMSOL MULTIPHYSICS v. 5.2, "WAVE OPTICS MODULE USER'S GUIDE", [Online]. Available: www.comsol.com. [Accessed 03 January 2021].

[25] H. P. Alves, J. F. Nascimento, E. Fontana , I. J. S. Coêlho and J. F. Martins-Filho, "Transition Layer and Surfac Roughness Effects on the Response of Metal based Fiber-Optic Corrosion Sensors", Journal of Lightwave Technology, vol. 36, no. 18, pp. 2597-2605, 2018.

[26] H. P. Alves, "Fibra Óptica de Perfil D: Fabrigação e Aplicações em Sensoriamento", Doctoral thesis (Doctoral thesis in Electrical Engineering) - UFPE, Recife, 2020.

[27] D. Marcuse, "Gaussian approximation of the fundamental modes of graded-index fibers", J. Opt. Soc, vol. 68, no. 1, pp. 103-109, 1978.

[28] A. A. Dias da Silva, H. P. Alves, F. C. Marcolino, J. F. do Nascimento and J. F. Martins-Filho, "Computational Modeling of Optical Fiber based Magnetic Field Sensors using Faraday and Kerr Magneto-Optic Effects", IEE Transactions on Magnetics, vol. 56, no. 9, pp. 1-9, 2020. 